International Journal of Food Science, Nutrition and Dietetics (IJFS)

ISSN 2326-3350

\title{
Food Safety Practice and Associated Factors of Food Handlers Working in Substandard Food Establish- ments in Gondar Town, Northwest Ethiopia, 2013/14
}

Gizaw $\mathrm{Z}^{1}$, Gebrehiwot $\mathrm{M}^{1}$, Teka $\mathrm{Z}^{2}$.

Research Article

${ }^{1}$ University of Gondar, College of Medicine and Health Sciences, Institute of Public Health, Department of Environmental and Occupational Health and Safety.

${ }^{2}$ University of Gondar, College of Natural and Computational Sciences, Department of Statistics.

\section{Abstract}

Introduction: Food borne illnesses comprise a broad spectrum of diseases and are responsible for substantial morbidity and mortality worldwide. The global incidence of food borne disease is difficult to estimate, but it has been reported that 2.1 million people died each year from diarrheal diseases and contaminated food contributes to 1.5 billion cases of diarrhea in children each year, resulting in more than three million premature deaths. In developing countries, up to an estimated $70 \%$ of cases of diarrheal diseases are associated with the consumption of contaminated foods. Approximately 10 to $20 \%$ of food-borne disease outbreaks are due to contamination by the food handler.

Objective: This study was conducted to assess food safety practices and associated factors of food handlers working in substandard food establishments of Gondar town, Northwest Ethiopia, 2013/14.

Methods: Institution based cross sectional study design was conducted to assess food safety practices and associated factors of food handlers. Four hundred three food handlers were taken randomly as study subjects and data were collected by observation by using standardized questionnaire and observational check lists. Ordinal logistic regression model was fitted to analyze the predictor variables.

Results: The overall level of food safety practices (good $-30.30 \%$, fair- $47.60 \%$ and poor $-22.10 \%$ ) was reported. Of a number of predictor variables analyzed age, marital status, service year, monthly income, food hygiene and safety training, attitude, knowledge and depth of knowledge were identified as factors affecting food safety practices.

Conclusion and recommendations: Compared to other similar studies, Low level of food safety practice (good - 30.30\%, fair- $47.60 \%$ and poor $-22.10 \%$ ) was reported. Therefore, Environmental health practitioners, the local Medias and the managers should do a lot to improve food safety practices of the food handlers. They should also design and implement food safety awareness creation programs.

Keywords: Food safety practice, food establishments, food handlers, ordinal logistic regression

\section{*Corresponding Author:}

Zemichael Gizaw,

University of Gondar, College of Medicine and Health Sciences, Institute of Public Health,

Department of Environmental and Occupational Health and Safety

E-mail:zemichael12@gmail.com

Received: July 11, 2014

Accepted: July 15, 2014

Published: July 25, 2014

Citation: Gizaw Z, Gebrehiwot M , Teka Z (2014)Food Safety Practice and Associated Factors of Food Handlers Working in Substandard Food Establishments in Gondar Town, Northwest Ethiopia, 2013/14. Int J Food Sci Nutr Diet. 3(7), 138-146. doi: http://dx.doi. org/10.19070/2326-3350-1400027

Copyright: Gizaw $\mathbf{Z}^{\odot}$ 2014. This is an open-access article distributed under the terms of the Creative Commons Attribution License, which permits unrestricted use, distribution and reproduction in any medium, provided the original author and source are credited.

\section{Introduction}

Food safety is an increasingly important public health issue to prevent or control food borne illnesses. Food borne illnesses comprise a broad spectrum of diseases and are responsible for substantial morbidity and mortality worldwide. In response to the increasing number of food borne illnesses, Governments all over the world are intensifying their efforts to improve food safety [1].

Food safety continues to be a public health problem worldwide because food borne illnesses are widespread. Consequently, consumers are increasingly concerned about food safety and quality; and demand more transparency in production and distribution [2]. The global incidence of food borne disease is difficult to estimate, but it has been reported that 2.1 million people died each year from diarrheal diseases. A great proportion of these cases can be attributed to contamination of food and drinking water [3].

In the world contaminated food contributes to 1.5 billion cases of diarrhea in children each year, resulting in more than three million premature deaths [4]. In South East Asia, approximately one million children under five years of age die each year from diarrheal diseases after consuming contaminated food and water [5].

In developing countries, up to an estimated $70 \%$ of cases of diarrheal diseases are associated with the consumption of contaminated foods [6]. Approximately 10 to $20 \%$ of food-borne disease outbreaks are due to contamination by the food handler [7].

Almost $75 \%$ of food borne illness outbreaks are assumed to be related to improper food handling practices by employees in food establishments [8]. The improper food handling practices have attributed by lack of adequate food safety knowledge. Thus, de- 
termining the level of food safety knowledge and practice and educating food establishment employees who handle food about proper food safety practices is crucial in preventing food borne illness outbreaks.

\section{Objectives of the study}

\section{General objective}

To assess food safety practices and associated factors of food handlers working in substandard food establishments in Gondar town, Northwest Ethiopia, 2013/14

\section{Specific objectives}

- To find out food safety practice of food handlers working in substandard food establishments

- To identify factors associated with food safety practice of food handlers working in substandard food establishments

\section{Methods and materials}

\section{Study design}

Institution based cross sectional study design was used to assess food safety practices and associated factors of food handlers working in substandard food establishments.

\section{Study area and period}

This study was conducted among food handlers working in substandard food establishments in Gondar town, Northwest Ethiopia, 2013/14

\section{Source population}

All food handlers working in substandard food establishments in Gondar town were source population.

\section{Study population}

Food handlers working in the randomly selected substandard food establishments were study population.

\section{Inclusion and exclusion criteria}

Inclusion criteria: Food handlers working in substandard food establishments and who had direct contact with food and food contact surfaces were included in the study.

Exclusion criteria: Food handlers who had not direct contact with food and food contact surfaces were excluded from the study.

\section{Sample size determination}

Single proportion formula was used to determine the sample size with the following assumptions.

- $\quad \mathrm{P}=0.5$ since there are no similar studies conducted in the area to assess food safety practice and associated factors of food handlers working in substandard food establishments.

- $\quad$ Margin of error $(\mathrm{W})=5 \%$

$95 \%$ confidence interval

$$
\begin{aligned}
\mathrm{n} & =(\mathrm{Za} / 2)^{2} \mathrm{pq} \\
& \mathrm{w}^{2} \\
& =384
\end{aligned}
$$

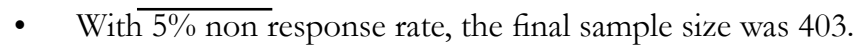

\section{Sampling method and procedure}

Systematic random sampling method was used to select the primary study units i.e. food establishments and simple random technique or lottery method was employed to reach the secondary study units i.e. food handlers.

\section{Data collection methods and instruments}

Data were collected from 403 food establishments and only one food handler was interviewed from on establishment. This was due to some food safety practices are practiced by the organization than the individual handlers. Data were collected by face to face interview and observation by using standardized questionnaire and observational check lists. The questionnaire had three sections: 1) general information such type of establishment, license statues, demographic information; 2) training and related information; 3) knowledge, attitude and practice towards food safety. Data on availability of equipments and facilities for hygienic practice were collected by an observation checklist. Critical elements to food safety and hygiene were listed on the observation checklist.

\section{Study variables}

\section{Dependent variables}

- $\quad$ Practice on food safety

\section{Independent variables}

- Sex,

- Age,

- Marital status,

- Educational status of the handlers,

- Person/s whom the handlers live together,

- Educational status of persons whom the food handlers live,

- Monthly income,

- Service year,

- Food safety information,

- Supervision by the owner or manager,

- Feedback from the customers,

- Food safety training,

- Knowledge on food safety

- Attitude on food safety

\section{Data quality assurance}

To ensure quality of data standardized questionnaire and check lists were developed from related published studies with little modification, training was given to data collectors and supervisors, the questionnaire was pretested and necessary correction was done after the pre test. The completed questionnaires were handled by the supervisors on each day of data collection. After checking for consistency and completeness, the supervisors had submitted the filled questionnaires to the principal investigator. Incorrectly filled or missed ones were sending back to data collectors for correction. Anything, which was unclear and ambiguous, 
was corrected on the next day of collection. The collected data were double entered to verify accuracy.

\section{Data management, processing and analysis}

Data were entered using EPI INFO version 3.5.3/2011 statistical software and were exported to SPSS version 20.0 for further analysis. Descriptive statistics of the collected data were done for most variables in the study using statistical parameters: percentages, means and standard deviations. Bivariate analysis will be used primarily to check which variables are associated with the dependent variable individually. Variables found to have association with the dependent variables will then analyze by ordinal logistic regression. Finally the variables which had significant association were identified on the basis of AOR, with $95 \%$ CI.

\section{Ethical considerations}

This study was carried out after getting permission from the ethical review committee of university of Gondar and the copy of permission letter was given for north Gondar administrative zone health bureau. Then, data were collected after getting written consent from the health bureau. Informed verbal consent was also obtained from each food establishments and study participants to conduct the study. Confidentiality was granted for information collected from each study participants. Participants' involve- ment in the study was on voluntary basis; participants who was unwilling to participate in the study \& those who wish to quit their participation at any stage was informed to do so without any restriction. Each respondent was informed about the objective of the study and privacy during interview was ensured.

\section{Results}

A total of 403 food handlers working in substandard food establishments were interviewed to assess their food safety practices and associated factors with $100 \%$ response rate. Data were collected on socio - demographic information; training and related information; knowledge, attitude and practice towards food safety.

\section{Socio demographic characteristics of respondents}

From the total respondents, 318(78.90\%) were female and $85(21.10 \%)$ were male with 1:3.74 male to female sex ratio. The mean age of the respondents was 23.53 years with standard deviation of 5.40 years $(23.53 \pm 5.40)$ and range 33 years $(15-48$ years). About $351(87.10 \%)$ of the respondents were Orthodox Christian. Two hundred twenty seven $(56.30 \%)$ of the respondents were attending secondary education and 306(75.90\%) were not married. The mean monthly income of the respondents was 484birr (table 1).

Table1.Socio - demographic characteristics of food handlers working in substandard food establishments in Gondar town, March, 2014

\begin{tabular}{|c|c|c|}
\hline Socio - demographic variables & Frequency & Percent \\
\hline \multicolumn{3}{|l|}{ Sex of the respondents } \\
\hline Male & 85 & 21.1 \\
\hline Female & 318 & 78.9 \\
\hline \multicolumn{3}{|l|}{ Age of the respondents } \\
\hline $15-17$ & 27 & 6.7 \\
\hline$>18$ & 376 & 93.3 \\
\hline \multicolumn{3}{|l|}{ Religion of the respondents } \\
\hline Orthodox & 351 & 87.1 \\
\hline Catholic & 2 & 0.5 \\
\hline Protestant & 18 & 4.5 \\
\hline Muslim & 32 & 7.9 \\
\hline \multicolumn{3}{|l|}{ Educational status } \\
\hline Illiterate & 45 & 11.2 \\
\hline Primary education $(1-8$ Grade $)$ & 105 & 26 \\
\hline Secondary education $(9-12$ Grade $)$ & 227 & 56.3 \\
\hline Higher education $(12+)$ & 26 & 6.5 \\
\hline \multicolumn{3}{|l|}{ Marital status } \\
\hline Single & 306 & 75.9 \\
\hline Married & 66 & 16.4 \\
\hline Divorced & 31 & 7.7 \\
\hline \multicolumn{3}{|l|}{ Monthly income } \\
\hline$<200$ & 29 & 7.2 \\
\hline $200-600$ & 307 & 76.2 \\
\hline$>600$ & 67 & 16.6 \\
\hline
\end{tabular}




\section{Types of establishment}

Data were collected from different food establishments found in Gondar town to assess food safety practices and associated factors. The type of establishments in which data were collected is presented below.

\section{Work experience}

To assess the contribution of work experience on food safety practices, data were collected about service year of the handlers.
The result is summarized by graph below.

\section{Food hygiene and safety information}

Out of the total food handlers interviewed about food hygiene and safety information and their source of information, 255 $(63.30 \%)$ have food hygiene and safety information and 148 $(36.70 \%)$ handlers have not any information. Source of information for food handlers who have food hygiene and safety information is presented below.

Figure 1. Types of establishments in which data were collected to assess their food safety practices and associated factors among food handlers working in substandard food establishments in Gondar town, March, 2014

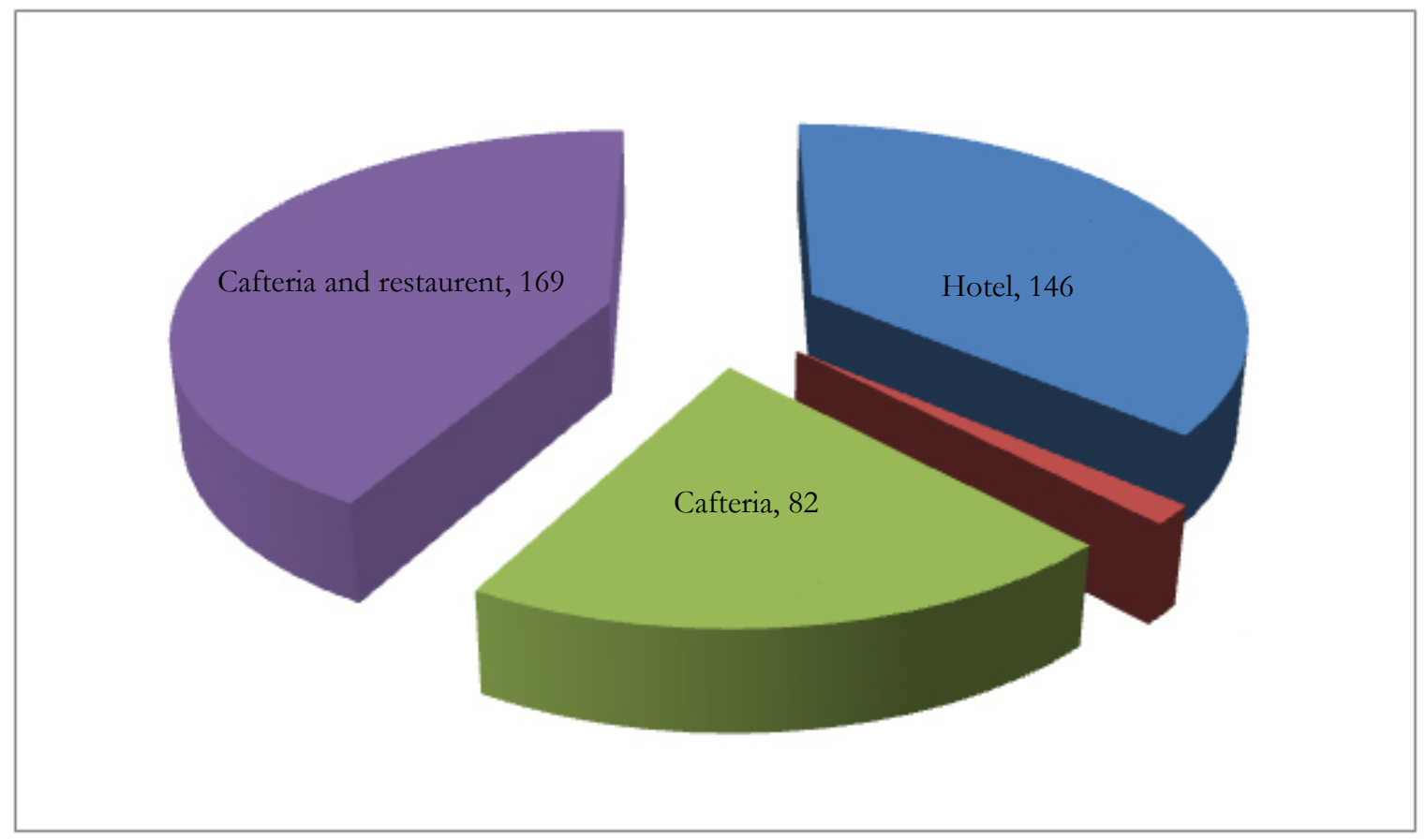

Figure 2: Work experience of food handlers working in substandard food establishments in Gondar town, March, 2014

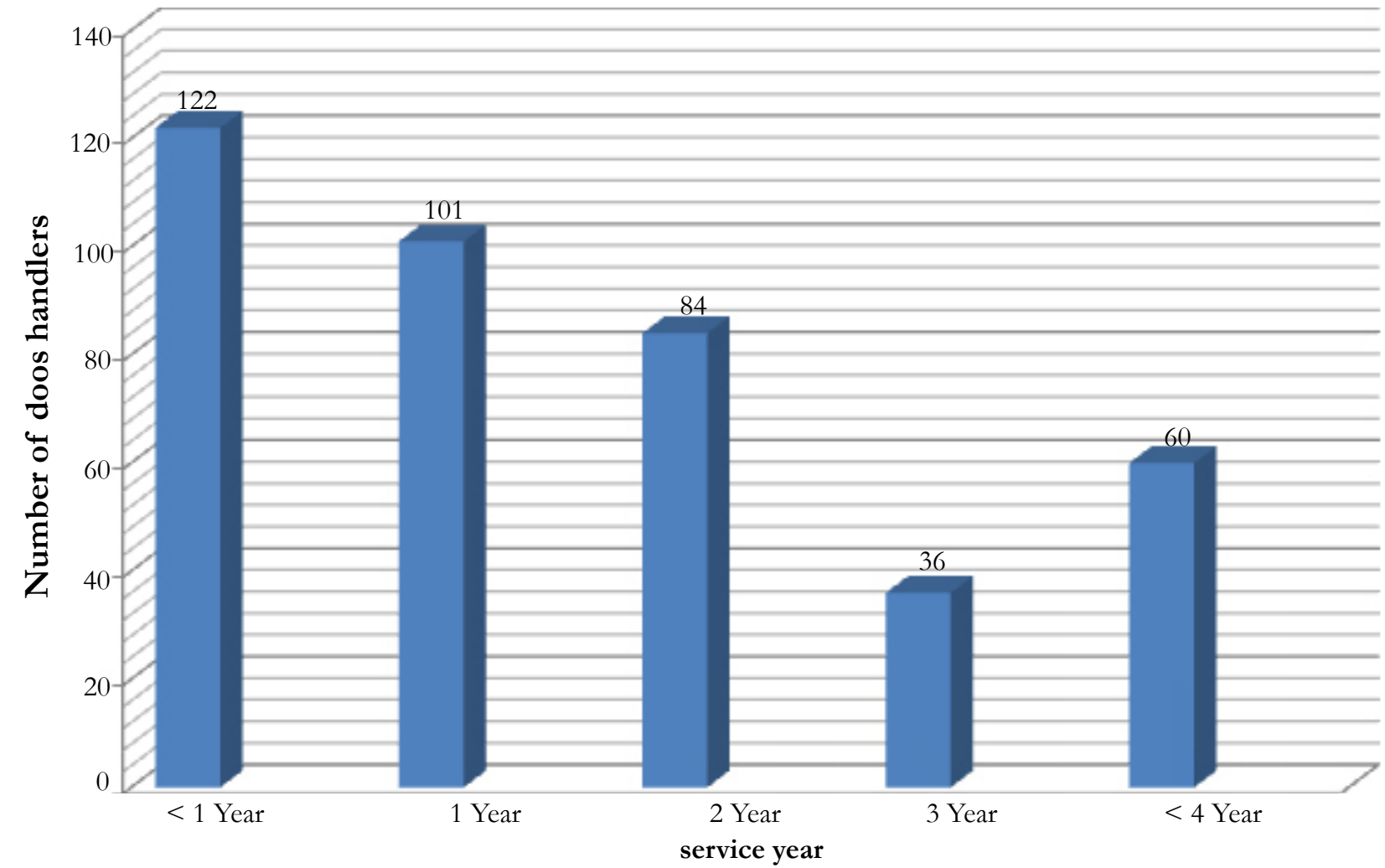


Figure 3: Source of food hygiene and safety information for food handlers working in substandard food establishments in Gondar town, March, 2014

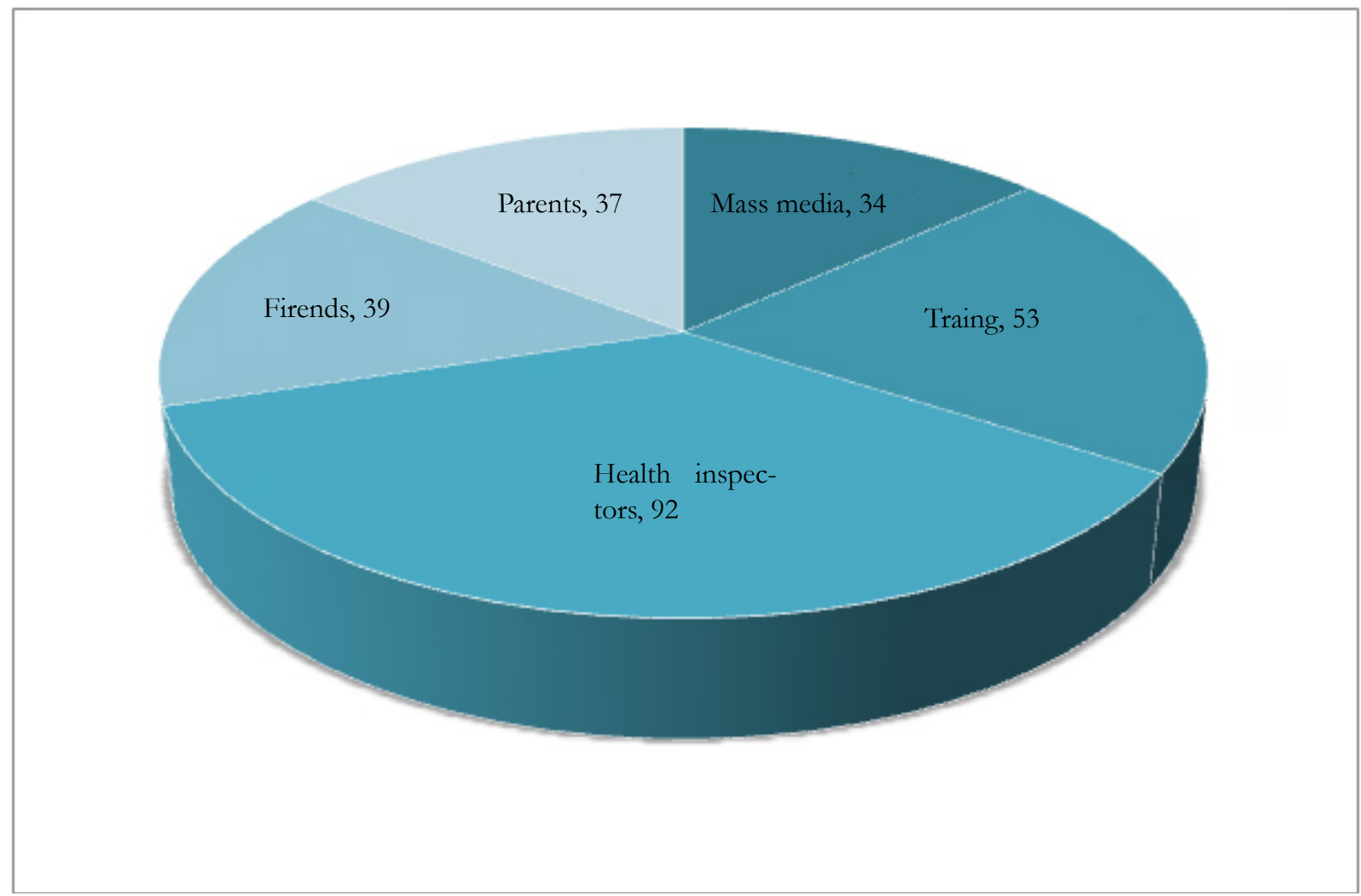

\section{Level of food safety practice}

Bloom's cut off points was used to determine practice level. To assess level of practice, 27 questions were prepared by using the CODEX food hygiene and safety principles. A score of 1 was given for each yes response and 0 for each no response. Level of practice was classified into poor level (Less than 59\%), fair level (60-80\%) and good level (80-100\%).

Similarly, the food safety knowledge and attitude were also measured as follow:

\section{Superficial knowledge}

Bloom's cut off points was used to determine knowledge level.
A total of 18 yes/ no questions were prepared by using the CODEX food hygiene and safety principles. A score of 1 was given for each correct response and 0 score for wrong response. Based on the sum scores level of knowledge was classified into Low level knowledge (Less than 59\%), Moderate level knowledge (60$80 \%)$ and High level knowledge (80-100\%).

\section{Attitude}

Attitude was assessed by 17 questions which were prepared from food hygiene and safety principles of CODEX and put on Likert's scale. The questions on Likert's scale had positive and negative responses that ranged from strongly agree, agree, neither agree nor disagree, disagree and strongly disagree. The scoring system used with respects to respondents' responses was as follows:

\begin{tabular}{|l|l|l|l|}
\hline Positive Statement & Negative Statement \\
\hline Choice & Scores & Choice & Scores \\
\hline Strongly agree & 4 & Strongly disagree & 0 \\
\hline Agree & 3 & Disagree & 1 \\
\hline Neutral & 2 & Neutral & 2 \\
\hline Disagree & 1 & Agree & 3 \\
\hline Strongly disagree & 0 & Strongly agree & 4 \\
\hline
\end{tabular}

The scores varied from 0 to 68 and all individual answers were summed up for total scores and calculated for means. The scores were classified into Positive Attitude (80\%-100\%), Neutral Attitude (60\%-80\%) and Negative Attitude (Less than 59\%) based on bloom's cut off points.

Therefore, based on the above measurements, level of food safety knowledge, attitude and practice of food handlers is sum- marized below (table 2).

Form 359 who have food safety knowledge (168 - Moderate level and 191 - High level), only 85 (23.68\%) of the handlers have deep knowledge and the rest have superficial knowledge. Deep knowledge was measured by seven analytical questions. The result is summarized below (table 3). 
Table 2. level of food safety knowledge, attitude and practice of food handlers working in substandard food establishments in Gondar town, March, 2014

\begin{tabular}{|l|l|l|}
\hline Variables & Frequency & \multicolumn{2}{l|}{ Percent } \\
\hline Level of superficial knowledge & \multicolumn{2}{l|}{} \\
\hline Low level & 44 & 10.9 \\
\hline Moderate level & 168 & 41.7 \\
\hline High level & 191 & 47.4 \\
\hline Level of attitude & \multicolumn{3}{|l|}{} \\
\hline Negative & 13 & 3.2 \\
\hline Neutral & 262 & 65 \\
\hline Positive & 128 & 31.8 \\
\hline Level of practice & \multicolumn{3}{|l|}{} \\
\hline Poor & 89 & 22.1 \\
\hline Fair & 192 & 47.6 \\
\hline Good & 122 & 30.3 \\
\hline
\end{tabular}

Table 3. Assessment result of deep knowledge on food safety of food handlers working in substandard food establishments in Gondar town, March, 2014

\begin{tabular}{|c|c|c|}
\hline $\begin{array}{l}\text { Questions which can asses depth of food safety } \\
\text { knowledge }\end{array}$ & Frequency & Percent \\
\hline \multicolumn{3}{|l|}{ List the 5 Keys to Safer Food } \\
\hline Those who can't list & 300 & 74.44 \\
\hline Those who can list $1-2$ keys & 55 & 13.65 \\
\hline Those who can list 3-4 keys & 48 & 11.91 \\
\hline Those who can list 5 keys & 0 & 0 \\
\hline \multicolumn{3}{|c|}{ How do you store large amounts of food in refrigerator? } \\
\hline Those who knew the correct storage & 145 & 36 \\
\hline Those who didn't know the correct storage & 258 & 64 \\
\hline \multicolumn{3}{|c|}{ How should dishes be washed to prevent food poisoning? } \\
\hline Those who knew the correct procedure & 160 & 39.7 \\
\hline Those who didn't know the correct procedure & 243 & 60.3 \\
\hline \multicolumn{3}{|c|}{ Where is the best place to store raw meat in the refrigerator? } \\
\hline Those who knew the storage place & 216 & 53.6 \\
\hline Those who didn't know the storage place & 187 & 46.4 \\
\hline \multicolumn{3}{|c|}{ For how long can you store meat and chicken in the refrigerator to eat later? } \\
\hline Those who knew the storage time & 73 & 18.11 \\
\hline Those who didn't know the storage time & 330 & 81.89 \\
\hline \multicolumn{3}{|l|}{ When should you wash your hands? } \\
\hline After visiting toilet & 37 & 9.2 \\
\hline Before and after preparation of foods & 117 & 29 \\
\hline After touching the skin, nose & 14 & 3.5 \\
\hline After I receive money & 5 & 1.2 \\
\hline Before washing of utensils & 98 & 24.3 \\
\hline During two or more of the above pick times & 132 & 32.8 \\
\hline \multicolumn{3}{|l|}{ What is the best way to wash your hands? } \\
\hline Those who knew the correct procedure & 187 & 46.4 \\
\hline Those who didn't know the correct procedure & 216 & 53.6 \\
\hline
\end{tabular}


Determinants of food safety practice of food handlers(table 4).

Table 4: Ordinal logistic regression of food safety practice with predictor variables of food handlers working in substandard food establishments in Gondar town, March, 2014

\begin{tabular}{|c|c|c|c|c|c|}
\hline \multicolumn{2}{|c|}{ Dependent variables and Predictor variables } & \multirow[t]{2}{*}{ Adjusted Odds ratio } & \multirow[b]{2}{*}{ Sig. } & \multicolumn{2}{|c|}{$\begin{array}{l}95 \% \text { C.I of the adjusted } \\
\text { AOR }\end{array}$} \\
\hline & & & & $\begin{array}{l}\text { Lower } \\
\text { Bound }\end{array}$ & $\begin{array}{l}\text { Upper } \\
\text { Bound }\end{array}$ \\
\hline & Level of practice & & & & \\
\hline \multirow[t]{4}{*}{ Dependent variables } & Poor & 1.616074 & 0.704 & 0.136422 & 19.1442 \\
\hline & Fair & 52.93157 & $0.002 *$ & 4.323218 & 648.7192 \\
\hline & Good [1] & - & - & - & - \\
\hline & Sex & & & & \\
\hline \multirow[t]{35}{*}{ Predictor variables } & Male & 1.26112 & 0.423 & 0.715338 & 2.223316 \\
\hline & Female [1] & - & - & - & - \\
\hline & Age & & & & \\
\hline & $15-17$ & 0.301194 & $0.011 *$ & 0.118837 & 0.764143 \\
\hline & $>18[1]$ & - & - & - & - \\
\hline & Educational status & & & & \\
\hline & Illiterate & 0.473786 & 0.207 & 0.148377 & 1.511345 \\
\hline & Primary education & 1.051271 & 0.923 & 0.385197 & 2.866237 \\
\hline & Secondary education & 1.304735 & 0.571 & 0.519962 & 3.273959 \\
\hline & Higher education [1] & - & - & - & - \\
\hline & Marital status & & & & \\
\hline & Single & 3.955077 & $0.021 *$ & 1.226298 & 12.75598 \\
\hline & Married & 1.253576 & 0.785 & 0.247338 & 6.347113 \\
\hline & Divorced [1] & - & - & - & - \\
\hline & With whom you live & & & & \\
\hline & Alone & 1.952284 & 0.064 & 0.960789 & 3.962995 \\
\hline & With my husband or wife & 2.091748 & 0.412 & 0.358796 & 12.19468 \\
\hline & With my sons or daughter (s) & 1.264909 & 0.746 & 0.306052 & 5.222624 \\
\hline & With my friend(s) & 1.336427 & 0.485 & 0.592147 & 3.019224 \\
\hline & With Parents [1] & - & - & - & - \\
\hline & $\begin{array}{l}\text { Educational status of persons } \\
\text { whom you live }\end{array}$ & & & & \\
\hline & Illiterate & 2.481839 & 0.113 & 0.806541 & 7.636963 \\
\hline & Primary education & 1.88891 & 0.17 & 0.761093 & 4.683286 \\
\hline & Secondary education & 0.935195 & 0.847 & 0.471423 & 1.855214 \\
\hline & Higher education [1] & - & - & - & - \\
\hline & $\begin{array}{l}\text { How long have you been work- } \\
\text { ing }\end{array}$ & & & & \\
\hline & $<1$ year & 1.707448 & 0.15 & 0.824482 & 3.536014 \\
\hline & 1 year & 1.624175 & 0.187 & 0.789781 & 3.340098 \\
\hline & 2 years & 0.960789 & 0.916 & 0.458865 & 2.01174 \\
\hline & 3 years & 3.370294 & $0.013 *$ & 1.294339 & 8.784598 \\
\hline & $>4$ years $[1]$ & - & - & - & - \\
\hline & Monthly income & & & & \\
\hline & $<200$ & 0.25487 & $0.007 *$ & 0.094137 & 0.690044 \\
\hline & $200-600$ & 0.635083 & 0.181 & 0.326606 & 1.234912 \\
\hline & $>600[1]$ & - & - & - & - \\
\hline
\end{tabular}




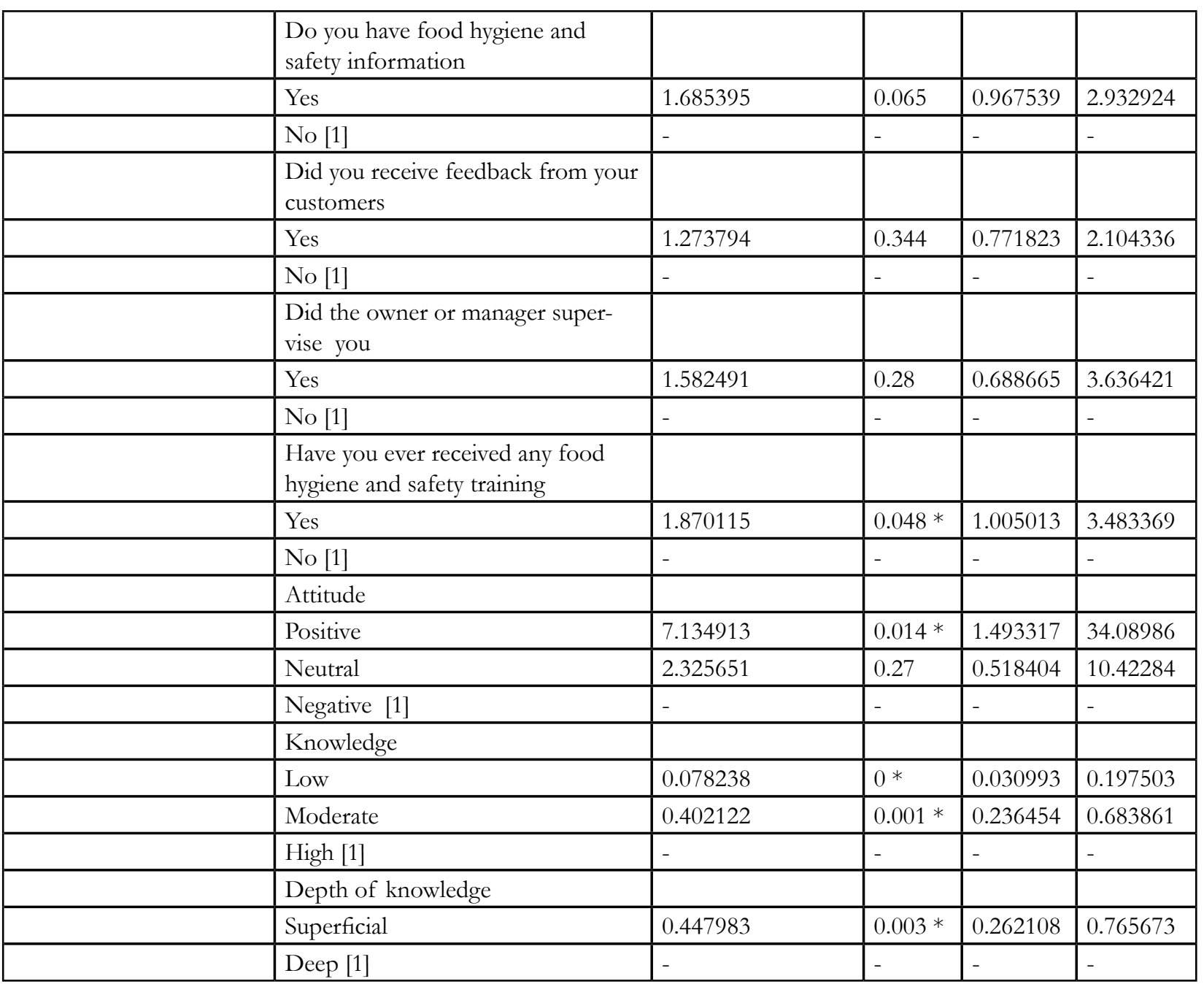

\section{Discussion}

\section{Level food safety practice}

This cross sectional study was conducted to determine food practices of food handlers working in substandard food establishments in Gondar town. Standardized questions or observation check lists were used to assess food safety practice. And Bloom cut off point was used to determine the level.

Accordingly, 192 (47.60\%) food handlers had fair food safety practices. The other $122(30.30 \%)$ and $89(22.10 \%)$ food handlers had good and poor level food safety practices respectively. This figure is markedly lower than levels reported by other studies and the figure also higher than other findings [9-12]. This may be due to the variation of educational status of the handlers and the provision of food hygiene and safety trainings.

\section{Associated factors with food safety practices}

The study also conducted to identify the associated factors of food safety practices of the handlers. This study revealed that age, marital status, service year, monthly income, food hygiene and safety training, attitude, knowledge and depth of knowledge were statistically associated with food safety practices. .

\section{Significant variables with food safety practices}

Age of the respondents was statistically associated with food safety practices. Those food handlers aged between 15 - 17 compared with 18 and above may have a chance to have either poor or fair level practices than good practices $(\mathrm{AOR}=0.3012,95 \%$ C.I $=$ $0.1188-0.7641)[13]$.

This study revealed that marital status was associated with food safety practices. Single handlers compared with divorced handlers had higher probability of good food safety practices (AOR = $3.9551,95 \%$ C.I $=1.2263-12.7540$ ) (14).

The current study showed that food safety practice was statistically associated with service years of the food handlers. Those food handlers who have been serving for three years compared with those who have been serving for more than three years were more likely to have good practice than poor or fair practices $(\mathrm{AOR}=$ 3.3703 , 95\% C.I $=1.2943-8.7846)$. This was also supported by other studies[13].

In this study, monthly income was the other statistically associated variable with food safety practice. Food handlers who earned 200 and below Ethiopian birr compared with who earned 600 and above may have a chance to have either poor or fair practice than good practice $(\mathrm{AOR}=0.2549,95 \%$ C.I $=0.0941-0.6900)$.

Like that of other studies, this study also d depicted that food safety training was significant variable. Food handlers who received food hygiene and safety training compared with handles who didn't receive training were more likely to have good food safety practice than poor or fair level $(\mathrm{AOR}=1.8701,95 \%$ C.I $=$ $1.0050-3.4834)[13,17]$. 
The result of the study has revealed that food safety practice is significantly related to the attitude of the workers. Food handlers who have positive attitude may have a chance to have good practice than poor or fair $(\mathrm{AOR}=7.1349,95 \%$ C.I $=1.4933-34.090)$ $[14,15]$.

Level of knowledge was also another statistically associated variable with food safety practice. Those food handlers who had low level knowledge than high level may have a chance to have either poor or fair practice than good practice (AOR $=0.0782,95 \%$ C.I $=0.0310-0.1975)$. This study also revealed that depth of knowledge was affecting the practice of food hygiene and safety. Food handlers with superficial knowledge compared to handlers with deep knowledge may have a chance to have either poor or fair practice than good practice $(\mathrm{AOR}=0.4480,95 \%$ C.I $=0.2621-$ $0.7657)[16,17]$.

\section{Conclusion}

Compared to other similar studies conducted in developed and developing countries, low level of food safety practice (good $30.30 \%$, fair- $47.60 \%$ and poor $-22.10 \%$ ) was reported.

Of a number of predictor variables analyzed age, marital status, service year, monthly income, food hygiene and safety training, attitude, knowledge and depth of knowledge were also identified as factors affecting food safety practices.

\section{References}

[1]. G.M. Subba Rao, R.V. Sudershan, Pratima Rao ,M. Vishnu Vardhana Rao (2007) Food safety knowledge, attitudes and practices of mothers-Findingsfrom focus group studies in South India. Science direct 49:441-449

[2]. P. T. Akonor, M. A. Akonor (2013) Food Safety Knowledge: The Case of Domestic Food Handlers in Accra. European Journal of Nutrition \& Food Safety 3(3): 99-111,

[3]. Fact Sheet $\mathrm{N}^{\circ} 23$. Food safety and food borne illness, revised January 2002http://www.who.int/.

[4]. World Health Organization (2002) Food Safety Programme "Food Safety An Essential Public Health Issue for the New Millennium".

[5]. Center for Science in the Public Interest. (2005) Food Safety around the World. Washington, D.C.

[6]. WHO (2008). Food borne Disease: A focus for Health Education.

[7]. Maizun MZ, Nyi NN (2002). Socio demographic characteristics and KAP of food handlers towards food sanitation in Malaysia. Southeast Asian j. trop. Med. and public health. 33(2)

[8]. Practical stuff - Food safety certification regulations in the United States. (2004). Journal of Environment Health 66(9):20.

[9]. Md Mizanur Rahman, Mohd. Taha Arif, Kamaluddin Bakar, \& Zainab bt Tambi. (2012) Food Safety Knowledge, Attitude And Hygiene Practices Among The Street Food Vendors In Northern Kuching City, Sarawak. Borneo Science 31.

[10]. George Amponsah Annor, Ekua Anamoaba Baiden. (2011) Evaluation of Food Hygiene Knowledge Attitudes and Practices of Food Handlers in Food Businesses in Accra, Ghana. Food and Nutrition Sciences 2:830-836 (http://www.SciRP.org/journal/fns).

[11]. Thidarat Cuprasitrut, Suwat Srisorrachatr, Duangjai Malai. (2011) Food Safety Knowledge, Attitude and Practice of Food Handlers and Microbiological and Chemical Food Quality Assessment of Food for Making Merit for Monks in Ratchathewi District, Bangkok. Asia Journal of Public Health $2: 1$

[12]. Penelope Tracy Campbell . (2011) Assessing the Knowledge, Attitudes and Practices of Street Food Vendors in the City of Johannesburg regarding Food Hygiene and Safety 11394:1827

[13]. Lerin Dirks, Dr. James Groves. (2010) Observation of Food Safety Behavioral Practices In Foodservice Employees After Training And Examination. University of Missouri-Columbia.

[14]. Laura R.Green. (2008) Behavioral Science and Food Safety. Direct from CDC Environmental Health Services Branch .Journal of Environmental Health 71(2):47-49

[15]. Jean Kennedy, Michelle Worosz, Ewen C. Todd, Maria K. Lapinski . (2008 ) Segmentation of US consumers based on food safety attitudes. British Food Journal 110 (7):691-705.

[16]. Brita Ball,Anne Wilcock, May Aung. (2010) Background Factors Affecting the Implementation of Food Safety Management Systems. Food Protection Trends, 30 (2): 78-86.

[17]. Yousouf Gaungoo, Rajesh Jeewone. (2013) Effectiveness of Training Among Food Handlers: A Review on the Mauritian Framework. Current Research in Nutrition and Food Science 1(1):01-09. 